\title{
Comportamiento a fatiga térmica de la capa de anclaje de nuevas barreras térmicas cerámicas obtenidas mediante proyección por plasma atmosférico
}

\author{
Thermal fatigue behavior of new ceramic thermal barrier coatings \\ obtained by the atmospheric plasma spray process
}

Comportamento à fadiga térmica da camada de fixação de novas barreiras térmicas cerâmicas obtidas mediante projeção por plasma atmosférico

Fecha de recepción: 14 de octubre de 2015

Pablo Carpio*

María Dolores Salvador-Moya*

Fecha de aprobación: 28 de febrero de 2016

Amparo Borrell ${ }^{\text {t*t }}$

Enrique Sánchez ${ }^{\text {***t }}$

\section{Resumen}

El desarrollo de nuevos materiales cerámicos para aplicarlos en recubrimientos protectores frente a la temperatura, también conocidos como barreras térmicas (Thermal Barrier Coatings o TBC), es de gran interés para muchos sectores, entre los que destacan las industrias de generación de energía, aeronáutica y automovilística.

En este trabajo se realizó un estudio del comportamiento a fatiga térmica, centrado en la respuesta de uno de los componentes que constituyen una TBC: la capa de anclaje. Los distintos componentes de la TBC fueron depositados mediante la técnica de proyección térmica por plasma atmosférico (Atmospheric Plasma Spraying o APS). Se estudió la microestructura y la composición de la capa de anclaje a lo largo de los ciclos térmicos de estudio.

Palabras clave: circona, fatiga térmica, proyección por plasma atmosférica, recubrimientos para barreras térmicas.

\footnotetext{
* Ph.D. Instituto de Tecnología de Materiales Universitat Politècnica de València (Valencia, España). Instituto de Tecnología Cerámica (ITC). Universitat Jaume I (Castellon, España). pabcarco@upv.es.

** Ph.D. Instituto de Tecnología de Materiales Universitat Politècnica de València (Valencia, España). dsalva@mcm.upv.es.

*** Ph.D. Instituto de Tecnología de Materiales Universitat Politècnica de València (Valencia, España). aborrell@upv.es.

*** Ph.D. Instituto de Tecnología Cerámica (ITC). Universitat Jaume I (Castellon, España). enrique.sanchez@itc.uji.es.
} 


\section{Abstract}

The development of new ceramic materials to protect the metallic components against corrosion and oxidation at high temperature, also known as thermal barriers coating (TBC), is of a great interest topic for many industrial and consumer sectors, such as energy generation, aeronautics and automotive industries.

This paper carried out a study on the thermal fatigue behavior of the bond coat, by centering the answer in one of the components which constitute a TBC: the anchor coat. The different TBC components were deposited by an Atmospheric Plasma Spraying (APS) Technique. Microstructure and anchor coat composition along the thermal cycles were studied.

Keywords: Atmospheric Plasma Spraying (APS), Thermal Barrier Coating (TBC), thermal fatigue, zirconia.

\section{Resumo}

O desenvolvimento de novos materiais cerâmicos para aplicá-los em recobrimentos protetores frente à temperatura, também conhecidos como barreiras térmicas (Thermal Barrier Coatings o TBC), é de grande interesse para muitos setores, entre os que destacam as indústrias de geração de energia, aeronáutica e automobilística.

Neste trabalho se realizou um estudo do comportamento à fadiga térmica, centrado na resposta de um dos componentes que constituem uma TBC: a camada de fixação. Os distintos componentes da TBC foram depositados mediante a técnica de projeção térmica por plasma atmosférico (Atmospheric Plasma Spraying ou APS). Estudouse a microestrutura e a composição da camada de fixação ao longo dos ciclos térmicos de estudo.

Palavras chave: fadiga térmica, projeção por plasma atmosférica, recobrimentos para barreiras térmicas, zircônia.

Cómo citar este artículo:

[1] P. Carpio, M. D. Salvador, A. Borrell and E. Sánchez, "Comportamiento a fatiga térmica de la capa de anclaje de nuevas barreras térmicas cerámicas obtenidas mediante proyección por plasma atmosférico", Fac. Ing., vol. 25 (42), pp. 67-73, may.-ago. 2016. 


\section{INTRODUCCIÓN}

Los recubrimientos de $\mathrm{ZrO}_{2}$ estabilizada con $\mathrm{Y}_{2} \mathrm{O}_{3}$ (YSZ) se utilizan, generalmente, como barreras térmicas (TBC, del inglés Termal Barrier Coating) en turbinas de gas. Estas barreras térmicas son sistemas complejos formados por diversas capas que sirven de protección al sustrato frente a la temperatura, la corrosión o el desgaste [1].

Cada una de las capas que forman la TBC tiene una función específica acorde con las propiedades del material. La capa de circona presenta una excelente resistencia al choque térmico, una baja conductividad térmica y un coeficiente de expansión térmica relativamente bajo [1]. La capa de anclaje, depositada entre el sustrato y la capa cerámica, consiste en una superaleación del tipo MCrAlY, cuya finalidad es disminuir la diferencia de contracción térmica entre el sustrato y la capa cerámica, además de actuar como barrera frente a la oxidación. Estas superaleaciones exhiben un perfil de propiedades que comprenden una buena ductilidad, alta resistencia térmica, buena resistencia a la corrosión y excelente resistencia a la oxidación [2]; sin embargo, los cambios microestructurales y las transformaciones de fase que tienen lugar durante prolongadas exposiciones a altas temperaturas han demostrado que afectan significativamente su comportamiento termomecánico y, por lo tanto, influyen en su vida útil [3].

Una de las técnicas más utilizadas para obtener una TBC es la proyección térmica por plasma atmosférico (Atmospheric Plasma Spraying -APS-) debido a su flexibilidad y bajo coste [4]. El polvo se inyecta en el interior de una pluma de plasma donde el material se funde y acelera hasta impactar con el sustrato, formándose una microestructura de gotas aplastadas o splats característica que componen el recubrimiento. Esta microestructura laminar que conforma los recubrimientos convencionales de APS puede contener numerosos defectos como poros, grietas o infundidos retenidos entre el material fundido [5].

En los últimos años, el empleo de nanopartículas en la obtención de recubrimientos se ha incrementado debido a la mejora que se produce en algunas propiedades [6]. En el caso de las barreras térmicas, a partir de nanopartículas se ha logrado reducir la conductividad térmica o aumentar la resistencia al choque térmico [7]. No obstante, las nanopartículas no pueden proyectarse directamente debido a su baja densidad y escasa fluidez. Una posible solución a este problema es aglomerar las nanopartículas en gránulos micrométricos antes de ser proyectados [5-7]. Hoy en día se pueden encontrar en el mercado diferentes polvos micrométricos nanoestructurados para ser depositados por APS sin dificultades.

En este trabajo se realizó un estudio del comportamiento en servicio de la capa de anclaje que forma parte de una TBC obtenida mediante APS. Se seleccionó una superaleación del tipo NiCoCrAlY, y como sustrato se empleó una aleación base titanio. Una vez obtenidas las TBC, se sometieron a ensayos de fatiga térmica y se caracterizaron microestructural y mecánicamente.

\section{EXPERIMENTAL}

\section{A. Obtención de las barreras térmicas}

El material seleccionado para el estudio como capa de anclaje es un polvo comercial de una superaleación de base Ni: Amdry 997 (Sulzer-Metco, Alemania), con composición Ni23Co20Cr8.5Al4Ta0.6Y. Como sustrato se empleó una aleación de titanio, Ti6Al4V, laminada en frío, con unas dimensiones de $4 \mathrm{~mm}$ de espesor por $100 \mathrm{~mm}$ de largo y $30 \mathrm{~mm}$ de ancho. Por último, se depositó un polvo comercial de circona estabilizada con itria (yttria stabilised zirconia, YSZ) sobre la capa de anclaje. El polvo de YSZ está compuesto por gránulos micrométricos nanoestructurados porosos (Nanox S4007, Inframat Corp., EEUU).

Tanto la capa de anclaje como la capa cerámica se depositaron mediante proyección térmica, empleando un equipo de proyección por plasma atmosférico, que consta de una antorcha monocátodo (F4-MB, Oerlikon-Metco, Alemania) guiada por un robot de 6 ejes (IRB 1400, ABB, Suiza). La capa de anclaje se depositó siguiendo las recomendaciones del fabricante (Tabla 1). Los parámetros de proyección de la capa cerámica se seleccionaron a partir de los resultados obtenidos en estudios previos [8]. 
Comportamiento a fatiga térmica de la capa de anclaje de nuevas barreras térmicas cerámicas obtenidas mediante proyección por plasma atmosférico

\section{TABLA 1}

PRINCIPALES PARÁMETROS DE PROYECCIÓN

\begin{tabular}{|c|c|c|c|c|c|c|}
\hline & Ar (slpm*) & $\mathbf{H}_{\mathbf{2}}$ (slpm*) & Intensidad (A) & $\begin{array}{c}\text { Distancia } \\
(\mathbf{m m})\end{array}$ & $\begin{array}{c}\text { Velocidad } \\
\mathbf{( m / \mathbf { s } )}\end{array}$ & $\begin{array}{c}\text { Caudal másico } \\
\mathbf{( g / \mathbf { m i n } )}\end{array}$ \\
\hline Bond coat & 65 & 8 & 650 & 145 & 1 & 40 \\
\hline YSZ & 35 & 12 & 600 & 100 & 1 & 45 \\
\hline
\end{tabular}

\section{B. Ensayo de fatiga térmica}

Los ciclos de fatiga térmica se llevaron a cabo en un horno eléctrico (Energon S.L) que cuenta con una plataforma ascendente/descendente, la cual permite el rápido calentamiento y enfriamiento de las muestras.
En la Fig. 1 se muestra una imagen del horno de fatiga y un detalle de la plataforma basculante, así como una gráfica de los ciclos de fatiga que se realizaron. Cada ciclo consiste en un calentamiento de las muestras a $900{ }^{\circ} \mathrm{C}$ durante 10 minutos, seguido de un rápido enfriamiento con aire frío. El número de ciclos estudiados fue de 20.

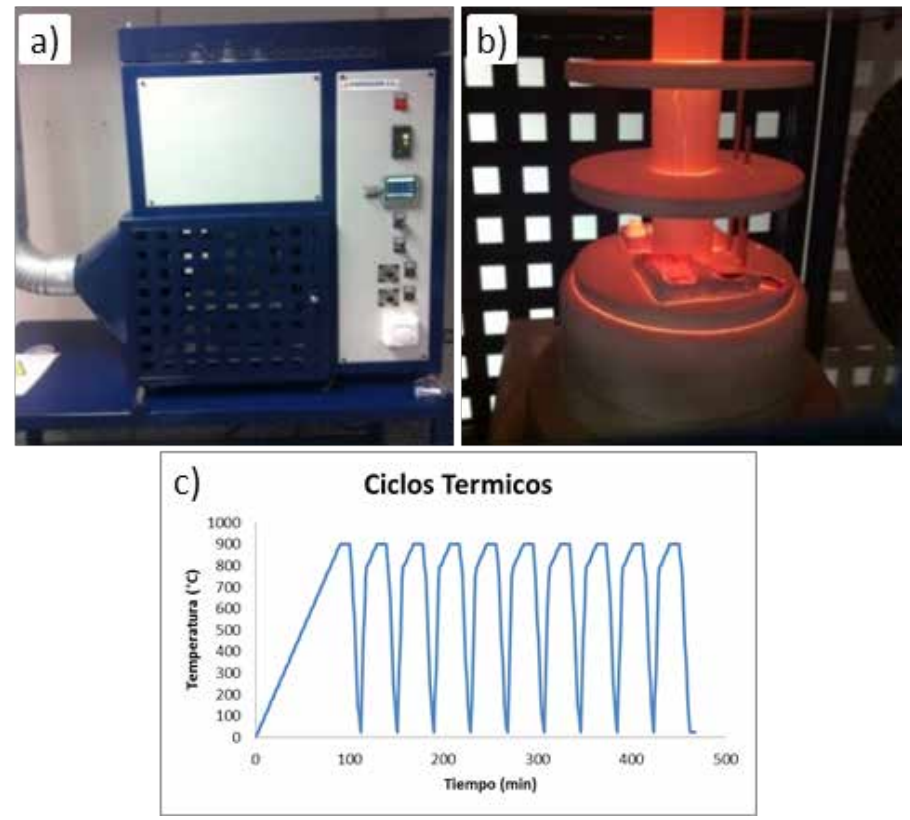

Fig. 1. a) Equipo de fatiga térmico empleado, b) detalle del mismo y c) secuencia térmica establecida.

\section{Caracterización de los recubrimientos}

La caracterización microestructural de los materiales se realizó con un microscopio óptico LV100 DA-U (Nikon, Japón) con software de imagen NIS-Elements junto con un microscopio electrónico de barrido (MEB) JSM 6300 (JEOL, Japón) con detector de rayos $\mathrm{X}(\mathrm{EDX})$. Se estudió la microestructura de los distintos recubrimientos antes y después de los distintos ciclos de fatiga térmica. Los espesores y la defectología de los recubrimientos se han determinado mediante análisis de imagen (Imagen Pro-Plus).

\section{RESUlTAdOS Y DISCUSIÓN}

En la Fig. 2.a se muestra la imagen MEB de la capa de anclaje proyectada sobre el sustrato. La capa de anclaje muestra una buena homogeneidad tanto en espesor como en morfología. Se puede observar claramente la microestructura tipo splats habitual en los recubrimientos obtenidos mediante una proyección térmica. El recubrimiento presenta una buena adherencia entre la capa de anclaje y el sustrato. En estudios anteriores se ha concluido que tamaños de splat pequeños disminuyen la conductividad térmica y mejoran la adherencia, debido a un mayor número de fronteras entre splats [9]. 

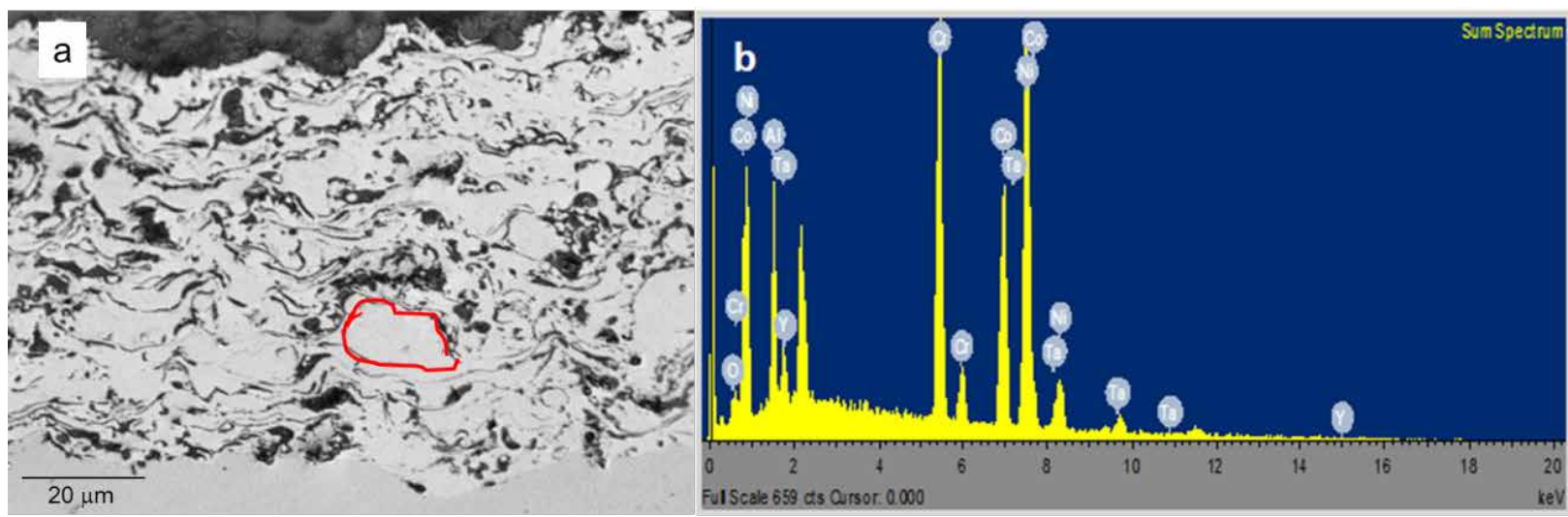

Fig. 2. a) Micrografía de MEB de la capa de anclaje AMDRY 997 proyectada con detalle de un splat y b) espectro composicional del splat seleccionado.

El microanálisis EDX de la capa de anclaje fue determinado en la zona fundida definida por un splat (Fig. 2). Los valores de EDX confirman la composición química suministrada por el fabricante (Tabla 2). Este hecho es importante, ya que indica que durante el proceso de proyección térmica no se ha generado una oxidación del material depositado.

\section{TABLA 2}

COMPOSICIÓN QUÍMICA OBTENIDA POR MICROANÁLISIS EDX DE LA CAPA DE ANCLAJE PROYECTADA

\begin{tabular}{|c|c|c|c|c|c|c|}
\hline Referencia & \multicolumn{6}{|c|}{ Composición química (\% peso) } \\
\hline $\begin{array}{c}\text { AMDRY } \\
997\end{array}$ & Ni & Co & Cr & Al & Ta & Y \\
& 43,3 & 22,9 & 20,2 & 8,5 & 5,0 & 0,6 \\
\cline { 2 - 7 } & & & & & & \\
\hline
\end{tabular}

En la Fig. 3 se muestra la capa intermedia que se ha generado entre la capa de anclaje y el sustrato como resultado de 20 ciclos térmicos. El microanálisis EDX muestra una difusión del aluminio en dos direcciones. Por un lado, se observa una ausencia casi total de este elemento en una región de 3-5 $\mathrm{mm}$ de espesor localizada en la capa intermedia formada; esta zona, que inicialmente pertenecía al sustrato, muestra una composición elemental de Ti y V, debido a la difusión del Al hacia la capa de anclaje. Por otro lado, se aprecia una mayor distribución de $\mathrm{O}$ y $\mathrm{Al}$ en los bordes de los splats de la capa de anclaje, lo que confirma la oxidación en el borde de grano. 
Comportamiento a fatiga térmica de la capa de anclaje de nuevas barreras térmicas cerámicas obtenidas mediante proyección por plasma atmosférico

\section{Capa de anclaje}

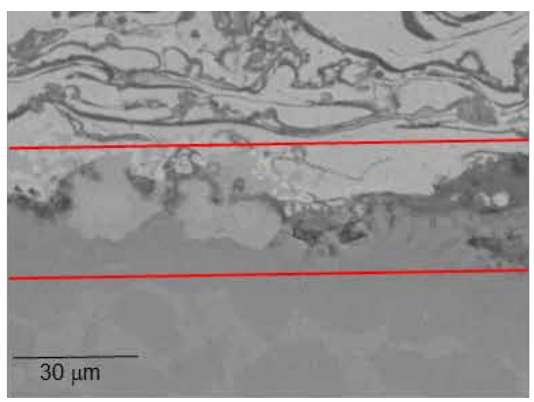

\section{Capa intermedia}

Sustrato

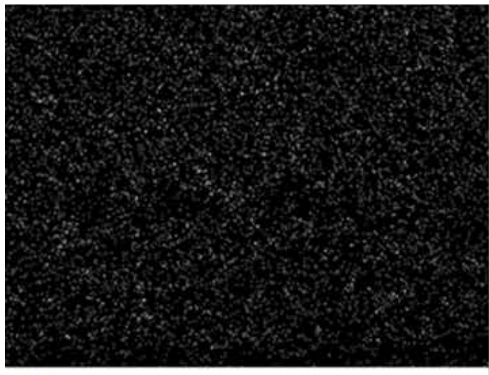

$\mathrm{OKa} 1$

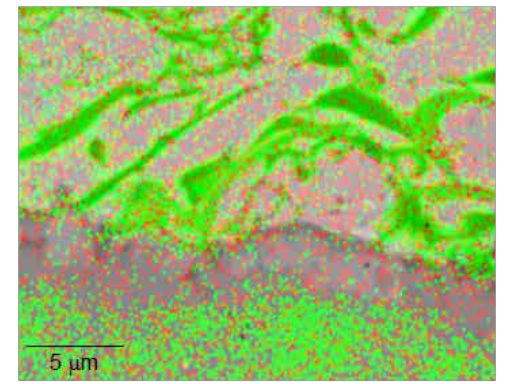

$5 \mu \mathrm{m}$

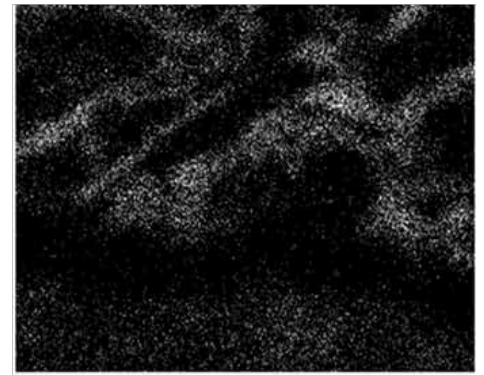

Al Ka 1

Fig. 3. Capa intermedia entre la capa de anclaje y el sustrato, generada tras 20 ciclos térmicos.

El análisis lineal de los elementos en la zona que comprende la interfase capa de anclaje/sustrato se muestra en la Fig. 4. En la Fig. 4.d se observa la difusión del Al desde el sustrato hasta la capa de anclaje, lo cual indica una alta compatibilidad entre los dos materiales empleados. Esta zona del sustrato, en la que la concentración de $\mathrm{Al}$ ha disminuido considerablemente, presenta ciertos puntos, no generalizados, en los que se observan inclusiones de $\mathrm{Co}$ y Ni; estos precipitados no deseados podrían generar fallos en la interfase capa de anclaje/sustrato, producir su desprendimiento y permitir el ataque de gases, con la consiguiente reducción del tiempo de servicio.
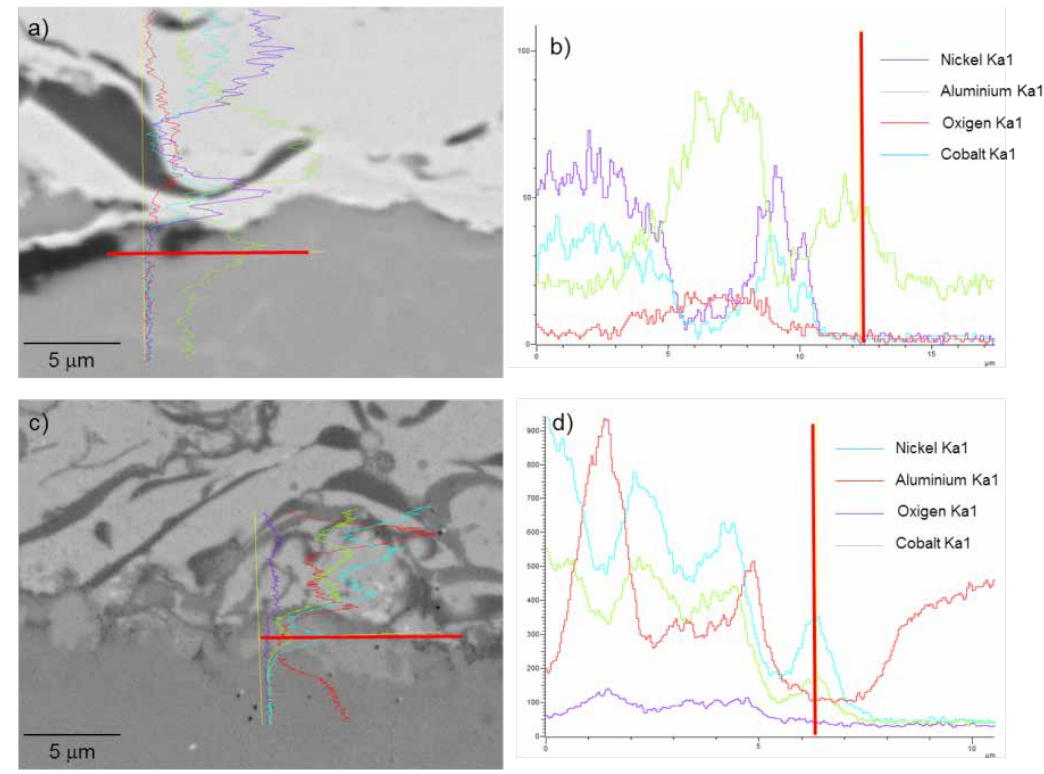

Fig. 4. Micrografías de MEB y análisis lineal de los elementos de la interfase capa de anclaje/sustrato en la muestra a) y b) de partida y c) y d), tras 20 ciclos térmicos. 


\section{Conclusiones}

Se estudió el comportamiento en servicio de una capa de anclaje del tipo NiCoCrAlY que conforma una barrera térmica. Para ello, el recubrimiento depositado por APS fue sometido a ciclos de fatiga térmica. La capa de anclaje mostró una alta compatibilidad con el sustrato de Ti6Al4V, así como una buena cohesión y adherencia, lo que garantiza la bondad del tiempo de servicio evaluado.

\section{Agradecimientos}

Este trabajo fue financiado por el Ministerio Español de Economía y Competitividad (MINECO), con fondos FEDER, a través de los proyectos MAT201238364-C03 y MAT2015-67586-C3-R. La Dra. Amparo Borrell agradece al MINECO su contrato de Juan de la Cierva, IJCI-2014-19839, y Pablo Carpio agradece su contrato predoctoral del Plan de Promoción de la Universitat Jaume I, acción 3.1 (Ref. PREDOC/2009/10).

\section{REFERENCIAS}

[1] W. Q. Wang, C. K. Sha, D. Q. Sun et al., "Microstructural feature, thermal shock resistance and isothermal oxidation resistance of nanostructured zirconia coating", Mater. Sci. Eng. A, vol. 424 (1-2), pp. 1-5, May. 2006. DOI: http://dx.doi.org/10.1016/j.msea.2005.10.025.

[2] N. K. Huang, "X-ray photoelectron spectroscopy studies on the surface of NiCoCrAlY coatings before and after high-temperature oxidation", Surf. Coat. Technol., vol. 53 (1), pp. 65-69, Jul. 1992. DOI: http://dx.doi.org/10.1016/02578972(92)90104-I.

[3] B. G. Mendis and K. J. Hemker, "Thermal stability of microstructural phases in commercial NiCoCrAlY bond coats", Scr. Mater, vol. 58
(4), pp. 255, Feb. 2008. DOI: http://dx.doi. org/10.1016/j.scriptamat.2007.10.016.

[4] J. M Guilemany. J. Fernández, J. Navarro et al., "Obtención de recubrimientos autolubricados por proyección térmica de plasma atmosférico (APS) como alternativa a los lubricantes líquidos", Bol. Soc. Esp. Ceram. Vidr., vol. 44 (4), pp. 211-214, Aug. 2005. DOI: http://dx.doi. org/10.3989/cyv.2005.v44.i4.376.

[5] P. Fauchais, G. Montavon, and G. Bertrand, "From Powders to Thermally Sprayed Coatings", Therm. Spray Technol., vol. 19 (12), pp. 56-80, Jan. 2010. DOI: http://dx.doi. org/10.1007/s11666-009-9435-x.

[6] M. Gel, E. H. Jordan, Y. H. Sohn et al., "Development and implementation of plasma sprayed nanostructured ceramic coatings", Surf. Coat. Technol., vol. 146-147, pp. 48-54, Sep. 2001. DOI: http://dx.doi.org/10.1016/S02578972(01)01470-0.

[7] R. S. Lima and B. R. Marple, "Thermal spray coatings engineered from nanostructured ceramic agglomerated powders for structural, thermal barrier and biomedical applications: A review", J. Therm. Spray Technol., vol. 16 (1), pp. 40-63, May. 2007. DOI: http://dx.doi. org/10.1007/s11666-006-9010-7.

[8] P. Carpio, E. Bannier, A. Borrell et al., "Influencia de los parámetros de proyección por plasma atmosférico en recubrimientos de YSZ obtenidos a partir de polvos micro y nanoestructurados", Bol. Soc. Esp. Ceram. Vidr., vol. 53 (4), pp 162-170, Aug. 2014. DOI: http://dx.doi.org/10.3989/cyv.202014.

[9] D. Schwingel, R. Taylor, T. Haubold et al., "Mechanical and thermophysical properties of thick PYSZ thermal barrier coatings: correlation with microstructure and spraying parameters", Surf. Coat. Technol., vol. 108-109, pp. 99-106, Oct. 1998. DOI: http://dx.doi.org/10.1016/ S0257-8972(98)00628-8. 